\title{
Nuclear Factor Erythroid 2-Related Factor 2
}

National Cancer Institute

\section{Source}

National Cancer Institute. Nuclear Factor Erythroid 2-Related Factor 2. NCI Thesaurus. Code C54255.

Nuclear factor erythroid 2-related factor 2 protein $(605 \mathrm{aa}, \sim 68 \mathrm{kDa}$ ) is encoded by the human NFE2L2 gene. This transcription factor is involved in cellular antioxidant defense and beta-globin activity modulation. 